\title{
An Overview of Techniques for Cardiac Left Ventricle Segmentation on Short-Axis MRI
}

\author{
Arseny Krasnobaev ${ }^{1 *}$ and Andrey Sozykin ${ }^{2}$ \\ ${ }^{1}$ Krasovskii Institute of Mathematics and Mechanics, Yekaterinburg, Russia \\ ${ }^{2}$ Ural Federal University, Yekaterinburg, Russia
}

\begin{abstract}
Nowadays, heart diseases are the leading cause of death. Left ventricle segmentation of a human heart in magnetic resonance images (MRI) is a crucial step in both cardiac diseases diagnostics and heart internal structure reconstruction. It allows estimating such important parameters as ejection faction, left ventricle myocardium mass, stroke volume, etc. In addition, left ventricle segmentation helps to construct the personalized heart computational models in order to conduct the numerical simulations. At present, the fully automated cardiac segmentation methods still do not meet the accuracy requirements. We present an overview of left ventricle segmentation algorithms on short-axis MRI. A wide variety of completely different approaches are used for cardiac segmentation, including machine learning, graph-based methods, deformable models, and low-level heuristics. The current state-of-the-art technique is a combination of deformable models with advanced machine learning methods, such as deep learning or Markov random fields. We expect that approaches based on deep belief networks are the most promising ones because the main training process of networks with this architecture can be performed on the unlabelled data. In order to improve the quality of left ventricle segmentation algorithms, we need more datasets with labelled cardiac MRI data in open access.
\end{abstract}

\section{Introduction}

Nowadays, heart diseases are the leading cause of death [1]. Non-invasive cardiac imaging technologies, such as magnetic resonance imaging (MRI), computerized tomography (CT), and echocardiography, are the essential tools for the diagnosis and treatment of such pathologies. In addition, with the help of these technologies the personalized heart models can be constructed in order to conduct the numerical simulations [2].

We present an overview of techniques for cardiac imaging segmentation, which is a delineation of heart chamber contours. The heart consists of four chambers, two ventricles and two atriums. Left ventricle (LV) is the biggest chamber of the heart and the most important one because it pumps blood to the entire body. Hence, in our review we are focused only on LV segmentation. Currently, the most precise source of information about the internal heart structure is the cine magnetic resonance imaging (MRI). We consider only the

$*$

Corresponding author: arseny.krasnobaev@,gmail.com 
short-axis MRI view because it is more widely used than the long-axis one. In addition, LV shape on the short-axis slices is much simpler, than on the long-axis, hence, most of methods use only short-axis data.

Segmentation of LV is a crucial step for estimation of the important diagnostics indicators because accurate LV contours provide significant amount of information about its shape and size. Usually, a procedure is performed on a series of short-axis slice images taken at equal time intervals throughout one cardiac cycle (4D MRI data). This is a spatio-temporal pattern recognition task.

At present, LV segmentation is usually performed manually because the automated methods do not meet accuracy requirements. Every LV segmentation algorithm faces the same challenges caused by the LV anatomical structure. Trabeculations and the papillary muscles are located inside the LV cavity and are almost indistinguishable from the myocardium by intensity. Therefore, many different algorithms tend to suffer from the same issue of inner contours shrinking (Fig. 1). Another common problem is that the LV cavity region sometimes appears to be very small on apical and basal slices that leads to drastic imbalance between number of pixels belonging to the LV region and to the background. Finally, variability in shape and intensity of LV among patients can be significant, especially for pathological cases. Despite these obstacles, fully automated algorithms have progressed significantly in past two decades.

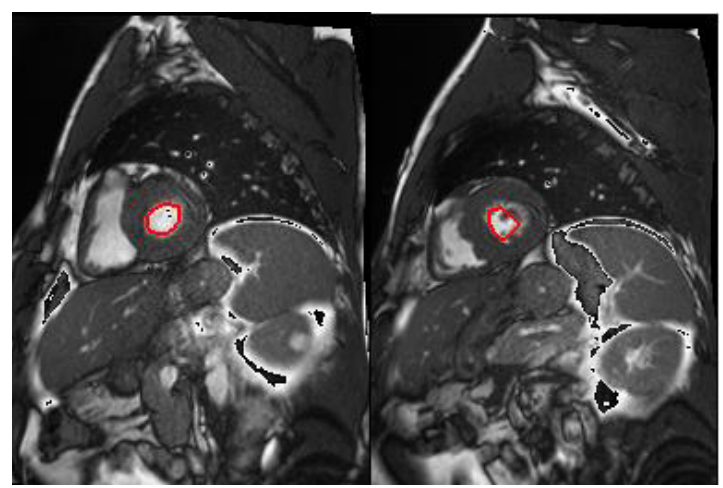

Fig. 1. The examples of LV segmentation on the short-axis MRI (left - accurate segmentation, right LV contour shrinked due to the papillary muscles).

\section{Segmentation Quality Evaluation}

One of the critical tasks of choosing the appropriate LV segmentation techniques is to determine the accuracy of the algorithms. Most popular approaches are based on usage of metrics that compare contours produced by the algorithm with the ground truth segmentation. Dice metric (DM) is a measure of similarity between regions:

$$
\operatorname{DM}(S, M)=2 \frac{|S \cap M|}{|S|+|M|},
$$

where $S$ is a detected region, $M$ is a manually labelled one. DM is the main tool for LV detection accuracy evaluation because it is simple and relevant.

Jaccard index is close to DM, but preserves triangle inequality:

$$
J(S, M)=\frac{|S \cap M|}{|S \cup M|}=\frac{D M(S, M)}{2-D M(S, M)}
$$


Hausdorff distance is another traditional approach to formalize the difference between regions in metric space:

$$
d_{H}(S, M)=\max \left(\sup _{s \in S} \inf _{m \in M} d(s, m), \sup _{m \in M} \inf _{s \in S} d(s, m)\right)
$$

In contrast to DM and Jaccard index, the Hausdorff distance considers metric distance between regions. Hence, it is sensitive to significant local shape deviations, but useless to detect continuous minor deviations like a contour bias. Therefore, DM and Hausdorff distance are complementary to each other and both of them should be used. The overall method reliability on a specific dataset can be formulated as a number of data items, on which quality metric exceeds some fixed threshold [3].

Finally, it is important to consider a specific dataset for quality evaluation, as image quality and anatomical deviations can greatly affect the results. There are a few cardiac MRI datasets publicly available:

- Sunnybrook Cardiac Data [4] was prepared for MICCAI (International Conference on Medical Image Computing and Computer Assisted Intervention) 2009 LV segmentation challenge. The dataset contains 4D MR images of 45 patients with ground truth contours of LV and myocardium. The dataset covers some anatomical pathologies.

- Kaggle Data Science Bowl Cardiac Challenge dataset includes short-axis MRI data for 700 persons. It is the biggest cardiac MRI dataset in an open access. Although the annotated contours are not provided, the dataset can be used for unsupervised learning.

- A dataset presented in the paper [5] provides short-axis MR images with annotated contours for 33 subjects.

\section{Left Ventricle Segmentation Techniques}

Although a large number of image segmentation algorithms has been developed, no universal solution suitable for every type of application is available. Hence, a wide variety of segmentation techniques has been developed specifically for various type of applications, including cardiac segmentation. Many completely different principles have been adopted, including heuristics and basic machine learning methods [6, 7], deep learning [8,9], deformable models [10], and random forests [11]. Segmentation was even treated as a tracking problem and several complex statistical models have been proposed [12].

Despite the significant differences in the algorithms, a number of general steps are common for most of the approaches. These steps form a kind of a framework for cardiac structure segmentation [13]. Usually, the first step is a pre-processing, such as noise removal and intensity standardization. Next, the region of interest (ROI) detection is performed. Since the short-axis slices contain not only the heart itself, but also a whole thorax, it is reasonable to separate ROI that includes the heart and minimum of unrelated pixels. This step improves both performance and accuracy of further segmentation. The best way to separate ROI is to use the fact that the heart is the only area that changes significantly within one cardiac cycle. There are several ways to do it, for instance, thresholding pixels by a standard deviation of their intensities over the time on middle slice [7]. Another approach [6] involves Fourier transform to compute the first harmonic, it uses not only the fact of changing intensity, but also its fundamental frequency. For supervised methods, above-mentioned steps are also applied for training data.

After pre-processing the segmentation is performed. Semi-automated approaches can include limited user interactions, i.e. selecting a point inside the LV cavity. 


\subsection{Deep Learning}

Convolutional neural networks (CNN) are deep artificial neural networks with a number of convolutional and subsampling layers [14]. Such architecture allows obtaining high node efficiency and reduces degree of freedom that lowers requirements for a number of training examples [15]. CNNs has become a preferred tool for general purpose 2D pattern recognition. Apparently, pure convolutional networks are rarely used for LV segmentation. A straightforward way is to train the deep network on ground truth contours to classify pixels [17]. Competitive results were declared, despite that only 2D segmentation was performed without using spatio-temporal relations among images.

Deep belief networks (DBN) are recently developed neural networks architecture. In contrast to CNN, DBN consists of fully connected layers, and the training process completely differs from the traditional backpropagation [16]. The training process is composed from two main stages. The first stage is an unsupervised pre-training of network treated as a stacked Restricted Boltzmann Machine, which is intended to learn unlabelled structure of data. The next step is a supervised stage of fine-tuning networks parameters with respect to labelled data with backpropagation. Such approach allows reaching high accuracy when a large amount of unlabelled data available to learn structure from, but only a small part of data is labelled. That makes DBN highly beneficial to real world applications. A recent research [9] has found an efficient way to combine DBN with level set method to attain a great endocardium segmentation quality.

\subsection{Random Decision Forest}

Random decision forest is a supervised regression and a classification machine learning method, known for its high generalizing ability and ease to train. Traditionally, the random forest is implemented by averaging results of a large number of decision trees, each of which is overfitted to perfectly recognize random subsampling or subspace of the original dataset. Such approach, called bootstrap aggregation, helps to overcome high predisposition of decision trees to overfitting and preserves high accuracy at the same time. Random forests are well-established for classification tasks, but relatively rarely used to image patter recognition. Nevertheless, a recent research has applied the combination of two forests for full spatio-temporal LV reconstruction using both the short-axis and long-axis view cine MRI images [11]. The approach uses one random forest for pre-processing (intensity standardization and orientation normalization) and another one for segmentation itself, with almost no other auxiliary processing. The relatively simple algorithm provides good results on the clear samples of interior slices. However, segmentation quality suffers on the apical and basal slices.

\subsection{Deformable Models}

Deformable models is a specific tool for image segmentation, based on the idea of iteratively evolving a curve or surface in order to minimize some functional [18]. An active contour model is a special case of deformable models, inspired by physics. This approach uses energy functional that is composed from image gradient throughout the curve evolved and contours tolerance for tension and bend. A level-set method is an implicit variation of the active contour model, which allows applying optimization directly to pixel grid, without necessity to parametrize a contour curve. The problem of active contour model is that all the terms required for object segmentation should be embedded into the energy function. Hence, to design such function manually is quite a challenging task. The contours that fitted to optimize simple gradient-based energy function tend to shrink inward or leak outward because such 
energy function reaches its minimum when it is on the ROI boundary. The problem can be solved by embedding into the energy function some prior information about LV shape, which can be obtained from another machine learning model. For instance, the energy can be regularized by the distance from the deformable contour to one retrieved by other approach, as described in [9]. Several recent researches, which declared state-of-art results (average DM value of 0.9-0.95), used combination of deformable models with another machine learning algorithms, such as DBN [9], CNN [8], or Markov random fields [10].

A combination of complex heuristics and deformable model, described in [19], preforms not as good as aforementioned combinations with advanced machine learning, yet it is quite competitive with DM value 0.91 on Sunnybrook dataset.

\subsection{Graph-based Methods}

According to the well-known image segmentation approach, which is based on max-flow problem solving, the image is treated as a graph. The nodes correspond to the pixels and the edges - to the pixel neighbourhood relations. The nodes are weighted with probability $a_{i}$ of corresponding to foreground, $b_{i}$ of corresponding to foreground and edges are weighted with $p_{i, j}$, which is a separation penalty. The goal is to find a partition of a pixel set into two sets, corresponding to background and foreground respectively by maximizing the functional:

$$
q(A, B)=\sum_{i \in A} a_{i}+\sum_{i \in B} b_{i}-\sum_{i \in A, j \in B} p_{i, j}
$$

The functional $q(A, B)$ is maximized by graph minimum cut, hence, the problem is reduced to finding max-flow/min-cut. A more general, but similar approach was used for LV segmentation [3] and reached DM of greater than 0.9 for LV cavity and 0.8 for myocardium segmentation.

\subsection{Basic Machine Learning and Heuristics}

Usually, the LV cavity is clearly distinguishable from the cardiac muscles on interior slices of short-axis MRI by intensity, thus it is possible to achieve the competitive results with only basic image segmentation algorithms and heuristics. Such approaches benefit from low requirements for computational power, which make them suitable for implementation in lowpower hardware. Recent research [6] has proposed a stack of efficient heuristics, which accurately segments most of the interior slices. Since segmentation is based on intensity thresholding, algorithm is prone to fail on the cluttered slices, but in most cases, it provides satisfactory results.

Another paper [7] relies on principle of component analysis over a number of hand-coded per-pixel features like gradient magnitude, intensity and median filter value, and k-nearest neighbours algorithm for pixel classification. The satisfying results were declared on a small private dataset.

\section{Conclusion}

Comparing the LV segmentation algorithms is a challenging task because published segmentation results was obtained in diverse conditions on different datasets using various quality metrics. The most popular among them are the Sunnybrook Cardiac Data and the dice metric (Table 1). Although most of the algorithms work well on the mid-ventricular slices, the quality of apical and basal slices segmentation still needs further improvements. 
Table 1. Comparison of LV cavity segmentation accuracy on the Sunnybrook cardiac data.

\begin{tabular}{|l|c|}
\hline \multicolumn{1}{|c|}{ Method } & Average dice metric \\
\hline CNN and deformable models [8] & 0.93 \\
\hline DBN and deformable models [9] & 0.90 \\
\hline Heuristics and deformable models [19] & 0.91 \\
\hline CNN standalone [17] & 0.86 \\
\hline
\end{tabular}

The current state-of-the-art approach is a combination of the deformable models with the advanced machine learning techniques, such as deep learning or Markov random fields. Such algorithms are being actively researched; the papers [8-10] declared segmentation accuracy comprising average dice metric value of $0.9+$ for inner and outer contours of LV on Sunnybrook dataset. We expect that DBN-based approaches are the most promising ones because the main training process of DBN can be performed on the unlabeled data.

Further development of segmentation techniques is impeded by the lack of training data. In order to develop the high quality fully automated LV segmentation algorithms we need more datasets with labelled MRI data in open access. In addition, the common approach to evaluation of cardiac segmentation algorithms quality is required.

This work was supported by the Russian Science Foundation (grant no. 14-35-00005).

\section{References}

1. D. Mozaffarian et al., Heart Disease and Stroke Statistics - 2016 Update. American Heart Association (2016)

2. S.F. Pravdin, V.I. Berdyshev, A.V. Panfilov, L.B. Katsnelson, O.E. Solovyova, V.S. Markhasin. Mathematical model of the anatomy and fibre orientation field of the left ventricle of the heart. Biomedical engineering online. 12:54 (2013)

3. I.B. Ayed, H. Chen, K. Punithakumar, I. Ross, Sh. Li, Max-flow segmentation of the left ventricle by recovering subject-specific distributions via a bound of the Bhattacharyya measure, Med. Image Anal., 16, (2012)

4. P. Radau, Y. Lu, K. Connelly, .G Paul, A.J. Dick, G.A. Wright. Evaluation Framework for Algorithms Segmenting Short Axis Cardiac MRI. The MIDAS Journal - Cardiac MR Left Ventricle Segmentation Challenge, http://hdl.handle.net/10380/3070, (2009)

5. A. Andreopoulos, K. John, Efficient and generalizable statistical models of shape and appearance for analysis of cardiac MRI, Med. Image Anal., 12(3), pp. 335-357, (2008)

6. X. Lin, B. Cowan, A. Young, Automated Detection of Left Ventricle in 4D MR Images: Experience from a Large Study, LNCS, 4190, pp. 728-735, (2006).

7. M.M.A. Hadhoud, M.I. Eladawy, A. Farag, F.M. Montevecchi, U. Morbiducci, Left Ventricle Segmentation in Cardiac MRI Images, American Journal of Biomedical Engineering, 2(3), pp. 131-135, (2012)

8. M. R. Avendi, A. Kheradvar, H. Jafarkhani, A Combined Deep-Learning and Deformable-Model Approach to Fully Automatic Segmentation of the Left Ventricle in Cardiac MRI, Med. Image Anal., 30, pp. 108-119, (2016).

9. T. A. Ngo, G. Carneiro, Left ventricle segmentation from cardiac MRI combining level set methods with deep belief networks, 2013 20th IEEE International Conference on Image Processing (ICIP), pp. 695-699, (2013)

10. L. Cordero-Grande, G. Vegas-Sánchez-Ferrero, P. Casaseca-de-la-Higuera, J. A. SanRomán-Calvar, A. Revilla-Orodea, M. Martín-Fernández, C. Alberola-López, 
Unsupervised 4D myocardium segmentation with a Markov Random Field based deformable model, Med. Image Anal. 15(3), pp. 283-301, (2011)

11. J. Margeta, E. Geremia, A. Criminisi, N. Ayache, Layered spatio-temporal forests for left ventricle segmentation from 4D cardiac MRI data, LNCS, 7085, pp. 109-119, (2011)

12. W. Sun, M. Cetin, R. Chan, V. Reddy, G. Holmvang, V. Chandar, A. Willsky, Segmenting and Tracking the Left Ventricle by Learning the Dynamics in Cardiac Images, LNCS, 3565, pp. 553-565, (2005)

13. T.N. Shewaye, Cardiac MR Image Segmentation Techniques: an overview, http://arxiv.org/pdf/1502.04252.pdf (2015)

14. L. Yann, B. Yoshua. Convolutional Networks for Images, Speech, and Time Series, The Handbook of Brain Theory and Neural Networks, MIT Press, pp. 1344, (1998)

15. M Lai, Deep Learning for Medical Image Segmentation, https://arxiv.org/pdf/1505.02000.pdf (2015)

16. G. E. Hinton, S. Osindero, Y.W. Teh, A fast learning algorithm for deep belief nets, Neural computation, 18(7), pp. 1527-1554, (2006)

17. P. V. Tran, A Fully Convolutional Neural Network for Cardiac Segmentation in ShortAxis MRI, http://arxiv.org/pdf/1604.00494.pdf (2016)

18. T. McInerney, D. Terzopoulos, Deformable Models in Medical Image Analysis: A Survey, Med. Image Anal. 1(2), pp. 91-108 (1996)

19. H. Hu,Z. Gao,L. Liu,H. Liu,j. Gao, et al. Automatic Segmentation of the Left Ventricle in Cardiac MRI Using Local Binary Fitting Model and Dynamic Programming Techniques. PLoS ONE 9(12) (2014) 